\title{
Identification of Volcano Hotspots by using Modified Standard Back Propagation (MBP) Algorithm via Satellite Images
}

\author{
Mr. S. Muni Rathnam ${ }^{1}$, Dr.T.Ramashri ${ }^{2}$ \\ ${ }^{I}$ (Department of ECE, SITAMS / JNTU Anantapur, India) \\ ${ }^{2}$ (Department of ECE, SVCE/SVU Tirupati, India)
}

\begin{abstract}
Remote Sensing is vital because of their pressing need due to the analysis of natural hazards. Among the various natural hazards, the Volcanoes are terrific hazard which may harm the nature as well as the living things. Due to the aforesaid reasons, the identification of volcanoes and their hotspot identification are important to protect the living things. A mechanism which will be utilized to identify the volcanoes and their hotspot from the satellite images is proposed. Initially, the color space of the satellite image will be converted to another color space to identify the contents of the image clearly. After this process, the image will be segmented to identify the volcano's hotspot. In order to identify the hotspot of volcano, the Artificial Neural Network (ANN) is utilized which uses Modified Standard Back Propagation (MBP) Algorithm. The proposed mechanism will be developed with the aid of the platform MATLAB (version 7.11).
\end{abstract}

Keywords: Artificial Neural Network(ANN), MBP, Remote sensing, Satellite Images and Volcano.

\section{Introduction}

The explosive growth of remote sensing technology, internet and multimedia systems poses great challenge in handling huge amount of data [5]. Advancement in the field of Remote Sensing has gone to an extent of taking the geospatial accuracy to few centimeters. Currently remote sensing has become a tool in the hands of scientific community to develop modeling applied in the projection right from natural disasters [4]. With the rapid development in remote sensing, digital image processing becomes an important tool for quantitative and statistical interpretation of remotely sensed images [3].

The use of remote sensing within the domain of natural hazards and disasters has become increasingly common, due in part to increased awareness of environmental issues such as climate change, but also to the increase in geospatial technologies and the ability to provide up-to-date imagery to the public through the media and internet [11]. One of the advantages of remote sensing is that the measurements can be performed from a great distance (several hundred or even several thousand kilometers in the case of satellite sensors), which means that large areas on ground can be covered easily. With satellite instruments it is also possible to observe, a target repeatedly; in some cases every day or even several times per day [12].

Nowadays, satellite imaging [6] is one of the most important sources of geographical, geophysical and environmental information [1]. Satellite images [7] are important source of information which is used in many environmental assessments and monitoring of agriculture, meteorology etc. [2]. They are important available data sources for map generation and updating of available maps. They provide accurate easily accessible and reliable spatial information for Geographical Information Systems [9]. The advanced technology where most satellite images are recorded in digital format virtually, all image interpretation and analysis involve some elements of digital processing [11]. Increasing use of satellite images which are remotely sensed images acquired periodically by satellites on different areas and for multiple purposes makes it extremely interesting for various applications [8].

\section{Related Researches: A Review}

A handful of researches are available and some of them are listed below. Mirnalinee Dhinesh et al. [12] have presented a multi-resolution based framework for detecting curvilinear structures from satellite images. Curvilinear structure detection finds its application in remote-sensed images for the extraction of networks such as roads, rivers, and highways. In the proposed methodology, curvilinear segments from the satellite images were extracted using multi-resolution GMM approach. The extracted curvilinear segments can be used for the detection and recognition of roads in the satellite images. Results have been showed the validity of the approach.

Debasish Chakraborty et al. [13] have discussed that the texture in high-resolution satellite images requires substantial amendment in the conventional segmentation algorithms. A measure has been proposed to compute the Holder exponent (HE) to assess the roughness or smoothness around each pixel of the image. The localized singularity information is incorporated in computing the HE. An optimum window size is evaluated so that HE reacts to localized singularity. A two-step iterative procedure for clustering the transformed HE image is 
adapted to identify the range of HE, densely occupied in the kernel and to partition Holder exponents into a cluster that matches with the range. Holder exponent values (noise or not associated with the other cluster) are clubbed to a nearest possible cluster using the local maximum likelihood analysis

Shwetank et al. [14] have discussed that the Digital image processing is collection of techniques for the manipulation of digital images by computer and its applications. This collection of methods in remote sensing is dominantly treated as Satellite Digital Image Processing (SDIP). A space borne Multispectral Image Processing System (MIPS) has been used since 1960 as a traditional satellite image processing system for data analysis and extraction of meaningful information from/in the earth surface. The MIPS system provides limited information due to the small number of spectral channels. Over the past two decades, advances in satellite imaginary system have made it possible for the collection of several hundred spectral bands for processing. This is commonly referred to as Hyper spectral Image Processing System (HIPS). Their study detailed the differences between MISP and HISP; and focused on the application of HIS for Rice crop-classification, plant growth, plant biophysical, biochemical, physiology properties in different spectral regions and their mapping.

Mohamed Awad [2] have discussed that the image segmentation is an essential step in image processing. The goal of segmentation is to simplify and/or to change the representation of an image into a form easier to analyze. Many image segmentation methods are available but most of these methods are not suitable for satellite images and they require a priori knowledge. In order to overcome these obstacles, a proposed technique in satellite image segmentation method has been developed using an unsupervised artificial neural network method called Kohonen's self-organizing map and a threshold technique. Self-organizing map was used to organize pixels according to grey level values of multiple bands into groups then a threshold technique is used to cluster the image into disjoint regions that proposed technique is called TSOM. Experiments performed on two different satellite images confirm the stability, homogeneity, and the efficiency (speed wise) of TSOM method with comparison to the iterative self-organizing data analysis method. The stability and homogeneity of both methods are determined using a procedure selected from the functional model.

Ashok et al. [15] have presented a GUI based multi spectral image enhancement used to achieve highly realistic and geo-scientifically corrects visualizations of real satellite imagery quite often the useful data in a digital image populates only a small portion of the available range of digital values. Image enhancement involved changing the original values so that more of the available range is used; this then increases the contrast between features and their backgrounds. It consists of reading the binary image on the basis of pixels taking them byte wise and displaying it, calculating the statistics of an image, automatically enhancing the color of the image based on statistics calculation using algorithms and working with RGB color bands. Finally the enhanced image has been displayed along with image histogram.

\section{Artificial Neural Network}

An Artificial Neural Network (ANN), often just called a neural network, is a mathematical model inspired by biological neural networks. A neural network consists of an interconnected group of artificial neurons, and it processes information using a connectionist approach to computation. The nodes can be seen as computational units. They receive inputs, and process them to obtain an output. This processing might be very simple (such as summing the inputs), or quite complex (a node might contain another network). The complexity of real neurons is highly abstracted when modeling artificial neurons. These basically consist of inputs, which are multiplied by weights, and then computed by a mathematical function which determines the activation of the neuron and another function computes the output of the artificial neuron. The idea of the back propagation algorithm is to reduce the error (difference between actual and expected results), until the ANN learns the training data.

The architecture of ANN is shown in fig (1) below where $i_{1}, i_{2}$ are the input values and $Y_{K}$ is the output value with $\boldsymbol{H}_{1}, \boldsymbol{H}_{2} \ldots \ldots \boldsymbol{H}_{N}$ is the hidden layer values.

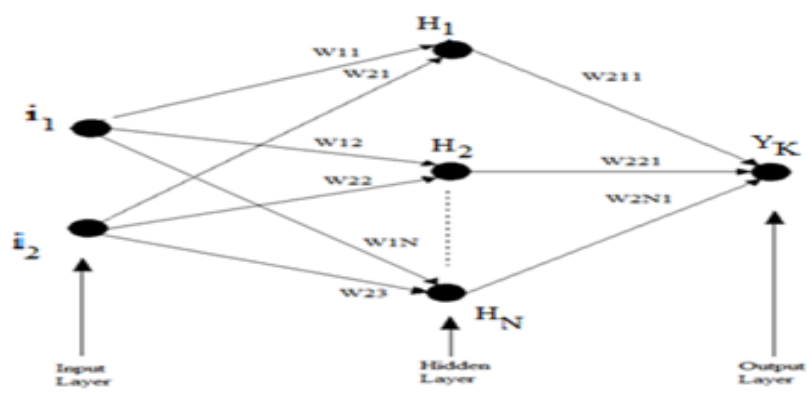

Fig1: Schematic representation of ANN 
The mathematical representation diagram for the ANN to find the output weightage of each node is shown in the fig (2).

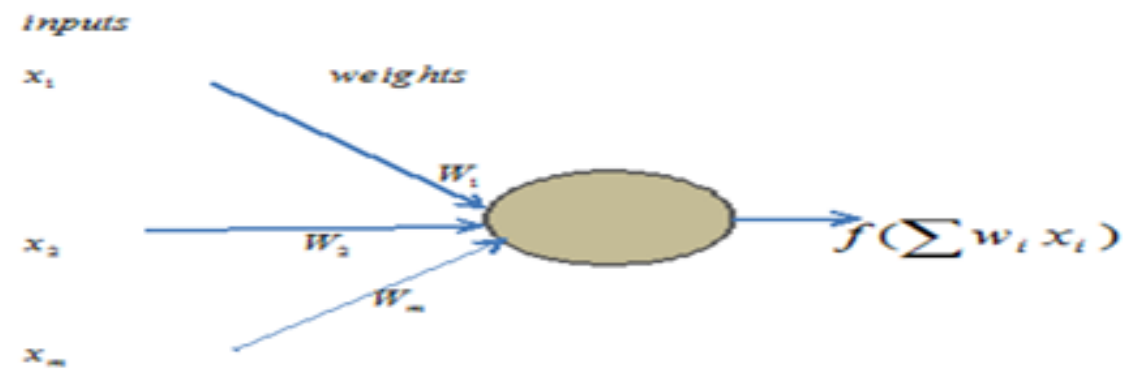

Fig 2: Mathematical representation of Artificial Neural Network

\section{Modified Standard Back Propagation (MBP) Algorithm}

MBP is a local adaptive learning scheme [16] which performs supervised batch learning in multilayer perceptrons. The learning algorithm is used for training a single layer perceptron. Let us consider a neural network with ' $n$ ' neurons in the input layer,' $j$ ' neurons in the hidden layer and one neuron in the output layer. Sigmoid activation function is used at both hidden and output layer. Jim Y.F Yam and Tommy W.S. Chow [17] Optimal Initialization is used.

Step1: Calculate the maximum and minimum value for all input variables $X_{i} ; i=1,2, \ldots \ldots . . n$.

Step2: Calculate the maximum distance between two points of the space

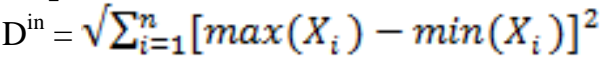

Step3: Calculate the $\mathrm{W}_{\max }$ to define the interval $\left[-\mathrm{W}_{\max }, \mathrm{W}_{\max }\right.$ ] from which the random weights for the hidden layer will be drawn. $\left.\quad \mathrm{W}_{\mathrm{nax}}=\frac{8.72}{D^{\text {in }}} \sqrt{(3} / n\right)$;

Step4: Evaluate the centre of the input space

$\mathrm{C}^{\text {in }}=\left[\left(\max \left(\mathrm{x}_{1}\right)+\min \left(\mathrm{x}_{1}\right) / 2,\left(\max \left(\mathrm{x}_{2}\right)+\min \left(\mathrm{x}_{2}\right) / 2\right)\right.\right.$, $\left.\left(\max \left(\mathrm{x}_{\mathrm{n}}\right)+\min \left(\mathrm{x}_{\mathrm{n}}\right) / 2\right)\right]$

Step5: Calculate the threshold value for hidden $\quad \mathrm{W}_{\mathrm{kh}}=-\sum_{i=1}^{n} C_{i}^{i n} \mathrm{w}_{\mathrm{ik}} ; \mathrm{k}=1,2, \ldots \ldots \ldots \ldots \ldots \mathrm{j}$.

Step6: Calculate $\mathrm{V}_{\max }$ to define the interval $\left[-\mathrm{V}_{\max }, \mathrm{V}_{\max }\right]$ for output layer $\quad \mathrm{V}_{\max }=\frac{15.10}{j}$;

Step7: Calculate threshold for output neuron $\mathrm{V}=-0.5 \sum_{i=1}^{j} V_{i}$

Step8: Create a feed forward neural network and initialize weights.

Step9: Calculate the net input to the hidden layer units and hidden layer output

Net input to hidden neurons $\mathrm{H}_{\mathrm{pk}}=\sum_{i=1}^{n} w_{i k}^{h} \mathrm{X}_{\mathrm{pi}} ; \&$ The output from the hidden layer $\mathrm{A}_{\mathrm{pk}}=\frac{1}{1+e^{-H_{p k}}}$

No. of training patterns $\mathrm{p}=1,2,3 \ldots \ldots \ldots$. And $\mathrm{w}_{\mathrm{ik}}{ }^{\mathrm{h}}$ weights between input and hidden layer.

Step10: Calculate the net input values and output of output neuron

The net input values $\operatorname{Net}_{\mathrm{p}}^{\mathrm{o}}=\mathrm{O}_{\mathrm{p}}=\sum_{k=1}^{j} w_{k}^{\circ} \mathrm{A}_{\mathrm{pk}} \&$ Output of neuron $\mathrm{Y}_{\mathrm{p}}=\frac{1}{1+e^{-O p}}$

Step11: Mean Square Error MSE $=\frac{1}{2}\left(d-Y_{p}\right)^{2}+$ MSE, initially MSE is zero;

$\mathrm{d}=0.75$ for volcano image and 0.25 for non volcano image

Step12: Calculate error for the output unit and hidden unit

Error for output unit $\delta_{\mathrm{p}}^{\mathrm{o}}=\left(\mathrm{d}-\mathrm{y}_{\mathrm{p}}\right)\left[\mathrm{y}_{\mathrm{p}}\left(1-\mathrm{y}_{\mathrm{p}}\right)\right]$

Error for hidden unit $\delta^{\mathrm{h}}{ }_{\mathrm{pk}}=\mathrm{A}_{\mathrm{pk}}\left(1-\mathrm{A}_{\mathrm{pk}}\right) \cdot \delta^{\mathrm{o}}{ }_{\mathrm{p}} \cdot \mathrm{w}_{\mathrm{k}}^{\mathrm{o}}$

Step13: Update weights on hidden and output layers

$\mathrm{W}_{\mathrm{k}}^{\mathrm{o}}(\mathrm{t}+1)=\mathrm{W}_{\mathrm{k}}^{\mathrm{o}}(\mathrm{t})+\eta \cdot \delta^{\mathrm{o}}{ }_{\mathrm{p}} \cdot \mathrm{A}_{\mathrm{pk}} ;$

$\mathrm{W}^{\mathrm{h}}{ }_{\mathrm{ik}}(\mathrm{t}+1)=\mathrm{W}^{\mathrm{h}}{ }_{\mathrm{ik}}(\mathrm{t})+\eta \cdot \delta^{\mathrm{h}}{ }_{\mathrm{pk}} \cdot \mathrm{X}_{\mathrm{pi}} ;$ Learning rate $\eta=0.1$ or 0.01 or 0.001

Weights updation is done for all images in the training patterns. This training process continued until Mean Square Error reaches minimum value[18]. 


\section{Image Recognition using MBP}

In this section, a mechanism which will be utilized to identify the volcanoes and their hotspot from the satellite images is proposed. Initially, the color space of the satellite image will be converted to another color space to identify the contents of the image clearly. After this process, the image will be segmented to identify the volcano's hotspot. In order to identify the hotspot of volcano, the Artificial Neural Network (ANN) is utilized. The neural network input can consist of pixels or measurements in images and the output can contain pixels, decisions, labels, etc. In this paper the neural network uses 6 inputs and 1 neuron in its output layer with 20 neurons in the hidden layer to identify the images. The database consists of normal satellite images and volcano images. For the image recognition, study the parameters of each image in the database. The six parameters used under this study are mean, variance, contrast, homogeneity, energy and correlation.

These parameters can be found out using the formulas below:

$$
\begin{aligned}
& \text { Mean }\left(\sigma_{M}\right)=\sum_{i, j} x(i, j) / n \\
& \text { Variance }\left(\sigma_{v}\right)=\frac{\sum x(i, j)^{2}}{n} \\
& \text { Contrast }\left(\sigma_{C}\right)=\sum_{i, j}|i-j|^{2} x(i, j) \\
& \text { Homogeneity }\left(\sigma_{H}\right)=\sum_{i, j} \frac{x(i, j)}{1+|i-j|} \\
& \text { Energy }\left(\sigma_{E}\right)=\sum_{i, j} x(i, j)^{2} \\
& \qquad\left(\sigma_{C R}\right)=\sum_{i, j}\left(i-\sigma_{M(i)}\right)\left(j-\sigma_{M(j)}\right) x(i, j) / \sigma_{V(i)} \sigma_{V(j)}
\end{aligned}
$$

Whereas $x_{i, j} \rightarrow$ is the intensity of pixels $i, j$ and $n$ is the no. of pixels

To create a network that can handle volcano images it is best to train the network in both satellite images and volcano images. To do this network will first be trained on satellite images until it has a least square. The above parameters for the each satellite images in the database are found out using the above equations. Then the network will again be trained on just satellite images. This ensures that the network will respond perfectly when presented with a normal satellite image.

Then the network is trained on volcano images and set a threshold value for instance $\lambda=0.5$. If an average of the parameter values of an image in the neural network is greater than or equal to the threshold value then the image is volcano image other else not. Then to identify the red spotted area from the volcano image intensity check process has to be done. This is performed using the RGB color representation of an image. Each color in the pixel has its own intensity value whereas in this volcano image representation Red (R), Green (G) and Blue (B) has its intensity value as 250,10 and 10 respectively. If the volcano image accumulates $\mathrm{R}$ value as greater than or equal to $250, \mathrm{G}$ and $\mathrm{B}$ as less than 10 , the image is identified as red spotted volcano image otherwise not. The flow chart representation for the above process is shown in the fig 4 . 


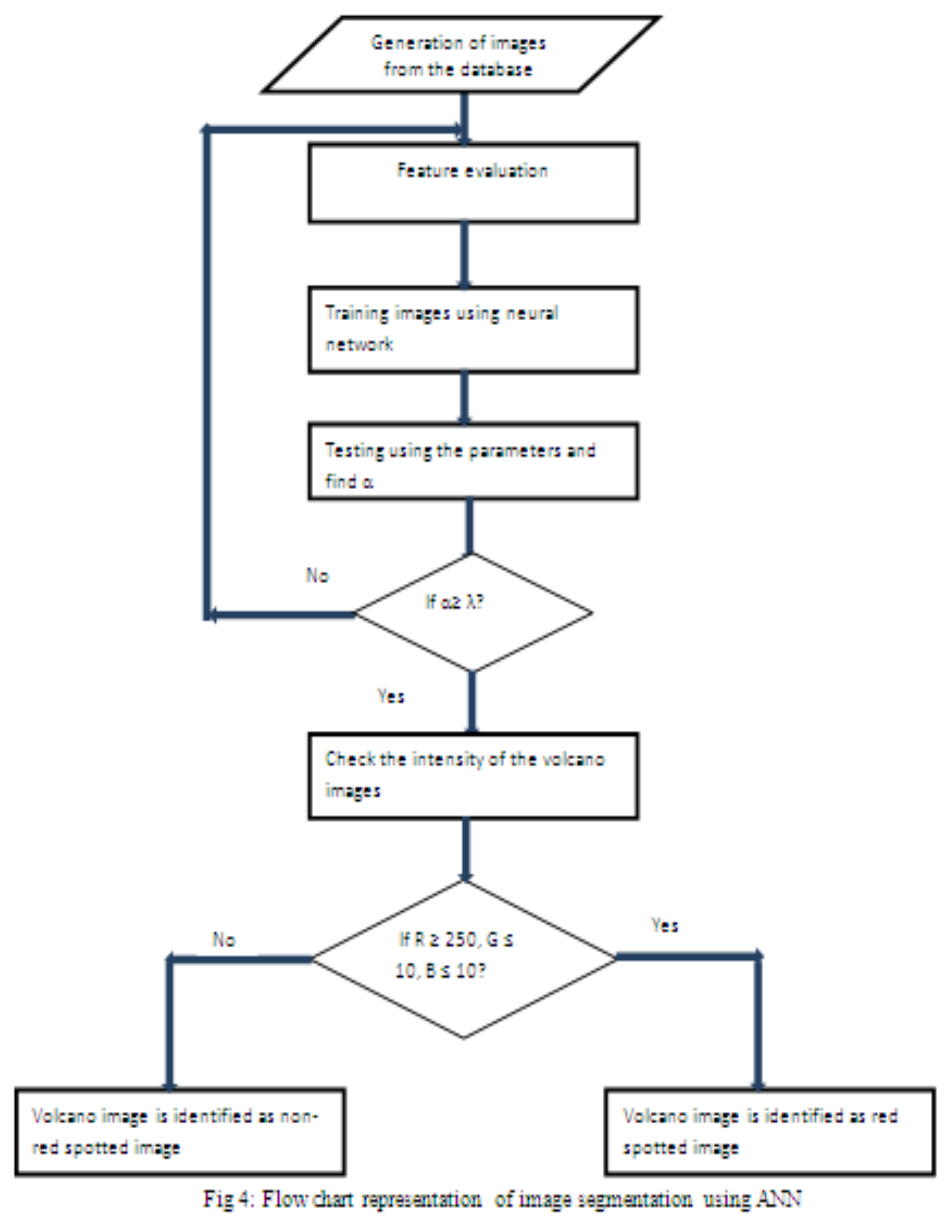

\section{Experimental Results}

The training of ANN is done by MATLAB software. In order to train neural network, selected features were evaluated; this evaluation was necessary to prevent non-uniform learning, in which the weight associated with some features converge faster than others. Initially, the color space of the satellite image will be converted to another color space to identify the contents of the image clearly. After this process, the image will be segmented to identify the volcano's hotspot. After segmentation a randomly chosen sample was divided into training, cross validation and testing datasets. The training data set was presented to the network for learning. Cross-validation is used to measure the training performance during training or stop training if necessary.

Testing data set is consisting of 100 satellite images in which 50 volcano and 50 non volcano images. Testing is done under the evaluation of sensitivity and specificity values. These values are among the terms true positive (TP), true negative (TN), false positive (FP), false negative (FN) [16].

The true positive, true negative, false positive and false negative values for the different datasets is shown in the table below

\begin{tabular}{|c|c|c|c|c|}
\hline & True Positive (TP) & $\begin{array}{c}\text { True Negative } \\
\text { (TN) }\end{array}$ & $\begin{array}{c}\text { False Positive } \\
\text { (FP) }\end{array}$ & $\begin{array}{c}\text { False Negative } \\
\text { (FN) }\end{array}$ \\
\hline Dataset & 41 & 42 & 08 & 09 \\
\hline
\end{tabular}

Table 1: True positive, true negative, false positive and false negative value

\section{Discussion}

Considering the values in the Table (1), sensitivity, specificity, accuracy, positive prediction value, negative prediction value, false positive rate, false discovery rate and Matthews's correlation coefficient can be found out. Sensitivity is defined as the ability to identify the volcano images and it is $82 \%$. The specificity, the ability to identify correctly the non volcano images which yields $84 \%$. Accuracy is the correct image recognition which is found as $83 \%$. The false positive rate, which is recognized as an error, has result of $16 \%$. Positive predictive value which is the proportion of the positive results has been identified as $83.67 \%$. The negative prediction value, the proportion of negative results, has the value $82.35 \%$. The false discovery rate which is the identification of false results and it is $16.326 \%$. The Matthew's correlation coefficient has been used for the 
identification of the results, which hold its value from the range of -1 to +1 . The value +1 concludes as a correct identification and vice versa. The value of Mathew's correlation is found as +0.66 . So it is concluded that volcano hotspot images can be easily identified by using Modified Standard Back Propagation (MBP).

\section{Conclusion}

We have presented a mechanism to identify the volcano hotspot images using Modified Back Propagation Algorithm (MBP). The training and testing of the dataset satellite images is undergone using crossvalidation and classification function (sensitivity and specificity measures) respectively. Results presented in this paper shows that red spotted in the volcano images is easily identified using Modified Standard Back Propagation (MBP). Even the results are encouraging image processing needed some special attention to improve the accuracy level where it is maximum of $83 \%$. Some adaptive techniques with the ANN will be used for the better improvement in the future works.

\section{References}

[1]. Starks and Kreinovich, "Multi-spectral inverse problems in satellite image processing", Systems Analysis Modeling Simulation, Vol.42, No.5, May 2002

[2]. Mohammad Awad, "An Unsupervised Artificial Neural Network Method for Satellite Image Segmentation", The International Arab Journal of Information Technology, Vol.7, No.2, pp.199-207, April 2010

[3]. Saheb Karim, Farah Riadh, Bassel Solaiman and Ben-Ahmed Mohamed, "Toward a multi-temporal approach for satellite image interpretation", The International Arab Journal of Information Technology, Vol.5, No.3, pp.281-287, July 2008

[4]. Sathiya, Vaidhiyanathan and Victor Rajamanickam, "Assessment of Ocean Parameters through the Satellite Images - AOOPSI", International Journal of Computer Theory and Engineering, Vol.1, No.4, pp.420-423, October 2009

[5]. Nigar Sulthana and Mahesh Chandra, "Image compression with Adaptive Arithmetic Coding", International Journal of Computer Applications, Vol.1, No.18, pp.31-34, 2010

[6]. Sadykhov, Dorogush, Pushkin, Podenok and Ganchenko, "Multispectral Satellite Images Processing For Forests And Wetland Regions Monitoring Using Parallel MPI Implementation", In proceedings of ENVISAT Symposium, Monteux, Switzerland, July 2007

[7]. Fleury, Self and Downton, "Multi-spectral Satellite Image Processing on a Platform FPGA Engine", In Military and Aeronautics Logic Devices (MAPLD’05), 2005

[8]. Ahmed Rekik, Mourad Zribi, Ahmed Ben Hamida and Mohammed Benjelloun, "An Optimal Unsupervised Satellite image Segmentation Approach Based on Pearson System and k-Means Clustering Algorithm Initialization", International Journal of Signal Processing, Vol.5, No.1, pp.38-45, 2009

[9]. Farnood Ahmadi, Valadan Zoej, Ebadi and Mokhtarzade, "Road Extraction from High Resolution satellite images using Image Processing Algorithms and CAD based Environments Facilities", Journal of Applied Sciences, Vol.8, No.17, pp.2975-2982,2008

[10]. Ahmed Rekik, Mourad Zribi, Ahmed Ben Hamida and Mohammed Benjelloun, "Review of satellite image segmentation for an optimal fusion system based on the edge and region approaches" IJCSNS, Vol.7,No. 10, pp.242-250,October 2007

[11]. Joyce, Belliss, Samsonov, McNeill and Glassey, "A review of the status of satellite remote sensing and image processing techniques for mapping natural hazards and disasters", Progress in Physical Geography, Vol.33, No.2, pp.183-207, 2009

[12]. Mirnalinee Dhinesh, Sukhendu Das, Koshy Varghese, "Automatic Curvilinear Structure detection from Satellite Images using Multi resolution GMM", International Journal of Imaging Science and Engineering,Vol.2,No.1,pp.154-157,January 2008

[13]. Debasish Chakraborty, Gautam Kumar Sen and Sugata Hazra, "High-resolution satellite image segmentation using Holder exponents", J. Earth Syst. Sci, Vol. ,NO.5,pp.609-617,October 2009.

[14]. Shwetank, Jain Kamal and Bhatia, "Review of Rice Crop Identification and Classification using Hyper- Spectral Image Processing System", International Journal of Computer Science \& Communication,Vol.1, No.1, pp.253-258, June 2010

[15]. Ashok and Rajan, "Multi Spectral Image Enhancement in Satellite Imagery", ACS-International Journal on Computational Intelligence, Vol.1, No.1, pp.13-20, Aug 2010.

[16]. V.V.Joseph Rajapandian and N. Gunaseeli “ Modified Standard Back Propagation Algorithm with Optimum Initialization for Feedforword Neural Network”, International Journal of Image Science and Engineering, Vol.1, No.3, Oct 2007.

[17]. Jim Y.F Yam and Tommy W.S. Chow "Feedforward Network Training Speed Enhancement by Optimal Initialization of Synaptic Coefficients" IEEE Transaction on Neural Networks, Vol.12, No.2, March 2001

[18]. S. Muni Rathnam and T. Ramashri "Identification of volcano hotspots by using standard back propagation (SBP) algorithm via satellite images" Journal of Theoretical and Applied Information Technology, Vol. 61. No.1 March 2014. 